\title{
VLC4WoT : Visible Light Communication for Web of Things
}

\author{
Mahmut Durgun, and Levent Gökrem** \\ Department of Mechatronic Engineering, Tokat Gaziosmanpasa University \\ Tokat, TURKEY \\ [e-mail: mahmut.durgun@gop.edu.tr, levent.gokrem@gop.edu.tr] \\ * Corresponding author: Levent Gökrem
}

Received May 9, 2019; revised August 15, 2019; revised October 6, 2019; accepted October 29, 2019; published April 30, 2020

\begin{abstract}
Recently, new devices have been developed for the Internet of Things concept. The devices commonly use RF (Radio Frequency) based wireless communication. With the increase in the number of devices, the space allocated for the radio frequency band in wireless communication fills rapidly. Visible Light Communication (VLC) is an alternative, secure and economical communication technology that uses light instead of radio frequencies. While Web of Things (WoT) is the adaptation of the experience and knowledge acquired from the web into the internet of things ecosystems. By combining these two technologies, the development of the Visible Light Communication for Web of Things (VLC4WoT) system, which can use VLC and WoT technologies, has been our motivation. In our study, microcontroller control circuit was created for VLC4WoT system. Control of the circuits over the internet was performed. VLC based receiver and transmitter units have been developed for wireless communication. Web based interface was created for control. The test apparatus consisting of four objects with four outputs and a transfer unit was carried out. In this test, communication was achieved successfully. It was presented in the study that VLC can be used in the web of things architecture. In the future, it is envisaged to use this system as a safe and economical system in indoor environments.
\end{abstract}

Keywords: Internet of Things, Visible Light Communication, Web of Things

The authors would like to thank to Tokat Gaziosmanpaşa University, Scientific Research Projects Comission (BAP) for supporting this study (Project number: 2017/83). 


\section{Introduction}

It is predicted that light will not only blink in the future but will also provide certain information to the objects around it [1]. This is called visible light communication (VLC). VLC, is a suggested IEEE 802.15.7 standard and accepted as a wireless communication method for Internet of Things (IoT) environment [2]. When compared with the current cable and wireless communications, VLC is an organic technology that is practical, secure because it only provides communication using the light in the environment; economic since it uses the led light that is currently used for lighting purposes and organic since it does not harm the environment and living beings [3], [4]. VLC communication is performed between the lighting unit which sends data and receivers that can make sense of visible light [2]. With using light in data transmission, VLC does not interact with existing radio frequency (RF) based communication [5]. Also using visible light spectrum in data transmission does not require a license to employ in communication [6].

Studies on machine to machine (M2M) communication gained further acceleration in accordance with the developments in mobile communication technologies [7]. In addition, systems that make life easier for people are required in many different areas that make use of wireless communication such as health, environment, transportation, structures, grids and cities which can be considered among M2M applications [8]. Today, technology giants along with the academia and entrepreneurs set off to design and produce internet objects as the internet of things increased in popularity among scientists and the industry [9]. We come across a new smart product almost everyday rapidly leading to new products and solutions developed as a result of this trend.

The IoT is the network of "things" or smart devices embedded with sensing, actuation, software, and network connectivity to sense and exchange data among the things, between the things, and with the outside world [10]. According to the estimations of Cisco, one of the leading companies in network technologies sector, it is expected that 50 billion objects in 2020 and 500 billion objects in 2030 will be connected to the internet and that the number of smart objects per person in 2020 will be 26 on average [11].

The development of IoT involves many issues such as infrastructure, communications, interfaces, protocols, and standards [12]. To universalization, studies are ongoing for ensuring that the IoT provides better solutions in two important topics. One of these is communication technologies and the other is framework studies. It is expected that the RF bands appointed to wireless mobile communication will be insufficient due to the communication between the developed objects and the rapid requirement for data in future [3]. It is predicted that VLC with a very wide and narrow band will be a proper solution for this problem as a new form of optic based communication that may provide an alternative to RF technology for the communication between objects [13].

Framework integrates IoT devices to the IoT ecosystem. The use of web technologies for this integration is gaining popularity [14]. This framework known as WoT is the inclusion of these framework objects into the World Wide Web (www) environment [15]. WoT architectures allow us to change the world in real time on the Web. WoT provides effective support for global communication, providing access to services and information. This allows different Web technologies to be used for more scalable and flexible development of applications [16]. The purpose of using the WoT architecture is to allow each device to be treated as components on the web using existing HTTP web standards [17]. Thus, devices can 
be easily integrated into web content [18], [19]. It allows you to manage with any device that can access the web, use internet services, access internet data sources, share via social media, and interact with devices connected to the internet.

In this study, we have focused on the internet objects using VLC technology, since there is a huge demand on higher bandwidth utilization, hence, on alternative technologies to RF based communication between internet objects. VLC provides larger bandwidth compared to RF technology, and it is more cost effective and safer for indoor environments. Moreover, VLC modules can be easily combined with existing lighting systems. Thus, the existing lighting systems can be easily modified to make the VLC system available at a low cost. Although VLC and WoT are known technologies, combining these two technologies to communicate internet objects with VLC in wireless communication and to control them in real time with a web-based user interface have not been considered and studied before. In this study, data in the cloud database are accessed over the internet via VLC for WoT (VLC4WoT) transmission unit. The acquired data are modulated and transferred to the light source. The receiving unit with the light sensor takes the data and makes the output either active or passive. Hence, a system has developed that makes use of the web of things framework and communicates via light in a rapid, secure and effective manner.

The main contribution of this study is presented below.

- Internet objects have been created that communicate with visible light instead of RF in wireless communication.

- Provided user comfort in management and monitoring by using web interface.

- Utilized high bandwidth for internet objects by means of communicating with light.

- Using of leds in the transmitter, photosensors in the receiver and software web significantly reduced the cost of the VLC4WoT.

- Created objects can be controlled in real time via web-based user interface.

This article is organized as follows. Section 2 briefly explains the work on VLC, IoT and WoT. In Section 3, we recommend the software and hardware architectures of the VLC4WoT platform. Section 4 introduces the experimental setup. In Section 5, we discuss the practice and experiment with performance analysis for the proposed platform. In Section 6, we finalize this article and explain future work.

\section{Related Work}

Light emitting diode (LED) and laser diode (LD) can be used as both light sources and transmitters for VLC. However, LEDs are preferred as majority transmitters because of their low cost, fast on-off time, high brightness level, low voltage operation and being in visible light band. LEDs are also preferred since they do not harm the human eye as it is observed in visible diodes [20]. Photodiodes and optical cameras can be used as receivers for the VLC. In general, two different types of photodiodes are used: PIN (Positive-Instrinsic-Negative) and APD (Avalanche Photo Diode). VLC is considered as the subtitle of Optical Wireless Communication (OWC) system. In these systems, the channel known as the environment through which data is transmitted is air [21].

VLC systems can be classified into outdoor VLC systems and indoor VLC systems. Car-to-car communication can be considered as an example for outdoor VLC systems [22]. As an other example, VLC systems can be applied to connect building to building or machine to 
machine where both the transmitter and receiver units can be mobile or fixed. The authors in [17] proposed a platform for traffic vehicles to communicate with each other or with traffic lights. In [23]-[25], since underwater communication through RF is difficult, the authors used VLC to have the underwater devices communicate with each other. Concepts such as smart home, smart building have become active discussion and research area. The use and rules of optical wireless communication in indoor environments are defined by the Elgala et al. [26], [27]. Millimeter waveform (60 GHz), wireless fidelity (WiFi) standard, VLC for high data rate communication have gained importance in internal communication of objects [28], [29]. The authors in [30] have created an object that can use the virtual reality with the information received from the lighting unit and provide information to the mobile phone to the camera phone. The authors proposed a model [31] in which smart home objects can be controlled using light, and receivers and transmitters are designed using LEDs. Researchers in [32], the internet connection and objects are shown to be provided with different colors and Wifi / VLC hybrid connection; however, information on the management of objects of the proposed system is not provided. IoT Device Management Protocol (IDMP) study has been performed for remote light management of IoT objects [33]. However, in this study, a wireless personal area network (WPAN) is required for accessing objects to access a special Java Virtual Machine (JVM) component and an illumination device for the IoT device.Numerous studies have been done on improving the performance of VLC in physical and MAC layers [24], [34]-[36]. As explained above, limited research has been done showing device management and data transfer in IoT networks using VLC [3].

For this reason, there is a need for a VLC system that can use the advantages provided by WoT to control objects in practice, which can be easily integrated into indoor applications. In this paper, we propose a framework for VLC-based IoT networks and also discuss device management, data transfer and transfer operations in IoT networks using VLC. Specifically, we discuss how to manage devices with VLC communication over the web and how to implement them together with the WoT architecture.

\section{VLC4WOT Platform Overview}

VLC4WoT platform has evaluated in two parts as hardware and software architecture. The details are discussed in the following.

\subsection{Hardware Architecture}

Fig. 1 shows a sample scenario related with the hardware structure of the VLC4WoT system. The hardware structure of the suggested system is comprised of three fundamental sections: (i) Cloud server that can store different types of data which can be accessed remotely via an Internet protocol (IP) address (ii) transmission unit that can transform the data it receives from the database into light data (VLC4WoTTx) (iii) receiving objects that can be used as web objects (VLC4WoTRx). 


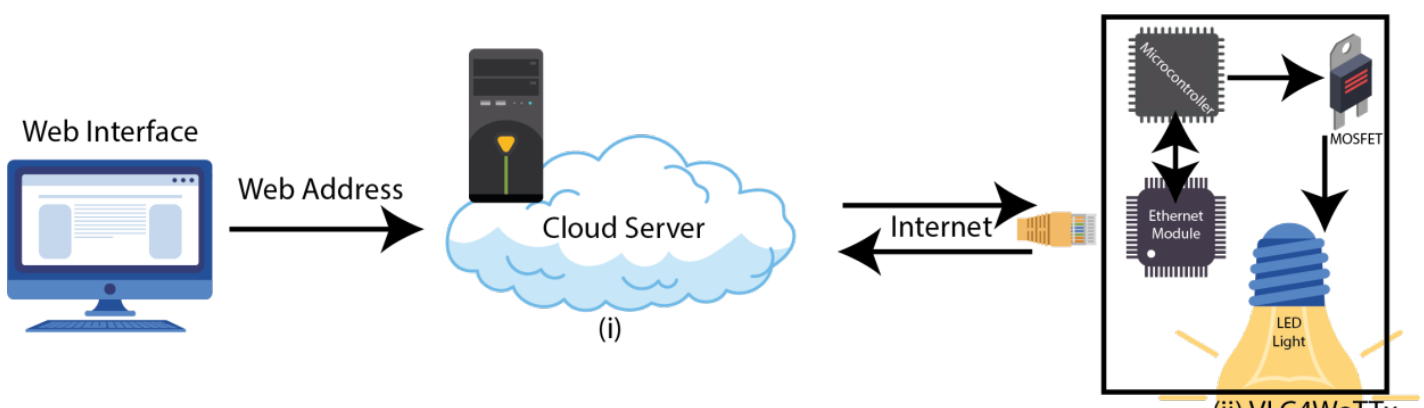

(ii) VLC4WoTTx

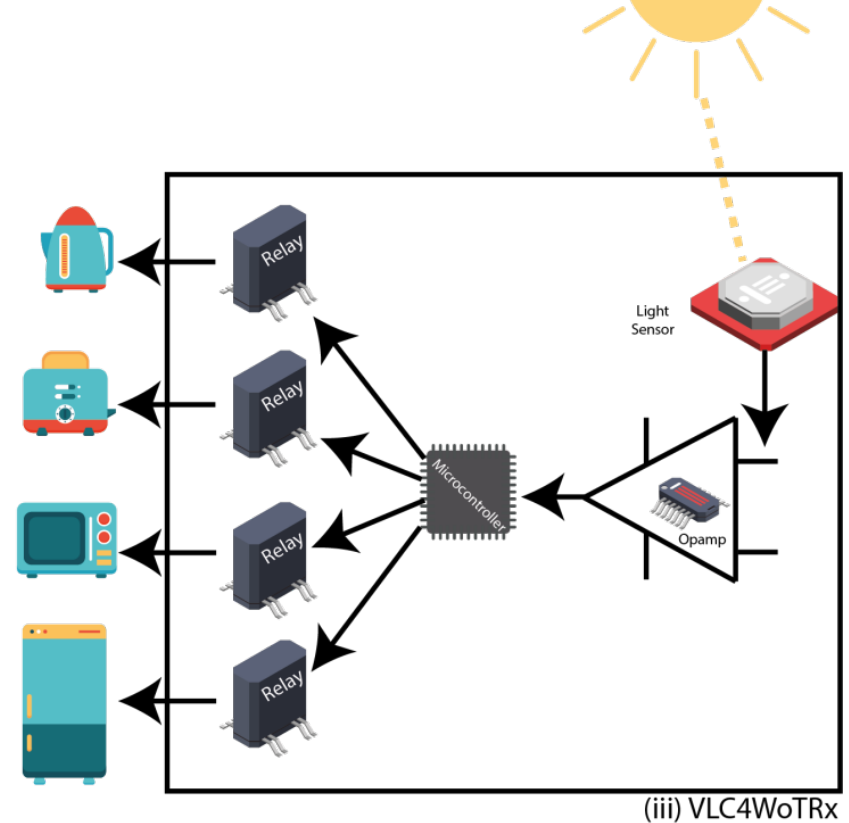

Fig. 1. Hardware Structure of VLC4WoT System.

\subsubsection{Cloud Server}

The cloud server is located in the center of a remote server. It is run as a virtual server. A processor for the prototype system is divided into 2 GB Ram, 50 GB harddisk space. Ubuntu Linux 14 operating system is installed on it.

\subsubsection{VLC4WoTTX}

The VLC4WoTTx object provides a connection to the cloud server and transmits its data by flashing the light. It consists of an 8 bit microcontroller, an Ethernet module for internet connection and MOSFET which can drive the LED lighting unit used in lighting. Microprocessor is ATMEGA328AU microcontroller of ATMEL. The microprocessor has 14 I/O pins. 6 of these pins have PWM outputs. By using pins with PWM, the light is blinking. The W5100 Ethernet module from WIZNET is used as the Internet connection. The Ethernet module supports 10M / 100M speed. Hardware supported TCP / IP support. Since the PWM output of the microprocessor is max $5 \mathrm{~V}$, MOSFET is used to drive the LED driver. IOR IRFZ44N with fast switching feature has used in our study. 


\subsubsection{VLC4WoTRx}

The VLC4WoTRx object is the receiving unit which detects the light changes via the photodiode located on it. With OPAMP, these changes are transferred to the microdentifier in order to be sensed. The meaningful data provides control of the relay circuit connected to the device. The control of household appliances connected to the relay is controlled according to their status. If the relay is on, the devices are off. Otherwise, the relay is off and the devices are on.

\subsection{Software Architecture}

The software architecture is comprised of the interface software developed for the user interface, server software, VLC4WoTTx software and VLC4WoTRx. The server has a static IP address. It operates on Linux operating system. LAMP (Linux + Apache + Mysql + Php) was installed on the server.

Fig. 2 shows the software architecture depicted as eight layers. The interface layer performs the control of the system on the web and the database server layer handles the database queries. The web server layer is the part where the server software is developed. The Representational State Transfer (REST) architecture establishes the communication with the objects, and the communication layer provides the communication infrastructure for the VLC4WoT components. The VLC4WoTTX Layer enables the light transmission of data from the cloud. The Channel is the spectrum of visible light band. The VLC4WoTRx layer comprises the objects that perceive light transmitted data and carry out the tasks involved. All the smart objects are real objects. The devices to be controlled are involved in this layer.

In order to operate the device, the user connects to the web address for a device. the state of the object is changed to run through the interface with the help of the button. As a result, the value of the corresponding id number is changed in the database table where the button is connected. A value of 1 is assigned for device operation and 0 for switching off the device. The VLC4WoTTx object connects to the server by using network access. Send HTTP requests to the server and check server responses. The request function takes in url and query, makes the request to the server, and returns the data on the page. The VLC4WoTTx object that reads this page reads the page. Assigns the values to read to the message variable. Analyzes the message content. Converts the message to numeric values. It transmits these values as logic information using the PWM output. Information is transmitted continuously as light. The VLC4WoTRx object constantly checks for light sensor changes. If the packet is properly captured, the message is decoded. Outputs are made active or passive according to the values by comparing the individual messages. Thus, the relay circuit becomes active or passive. According to this study, the objects in the environment are turned on or off. 


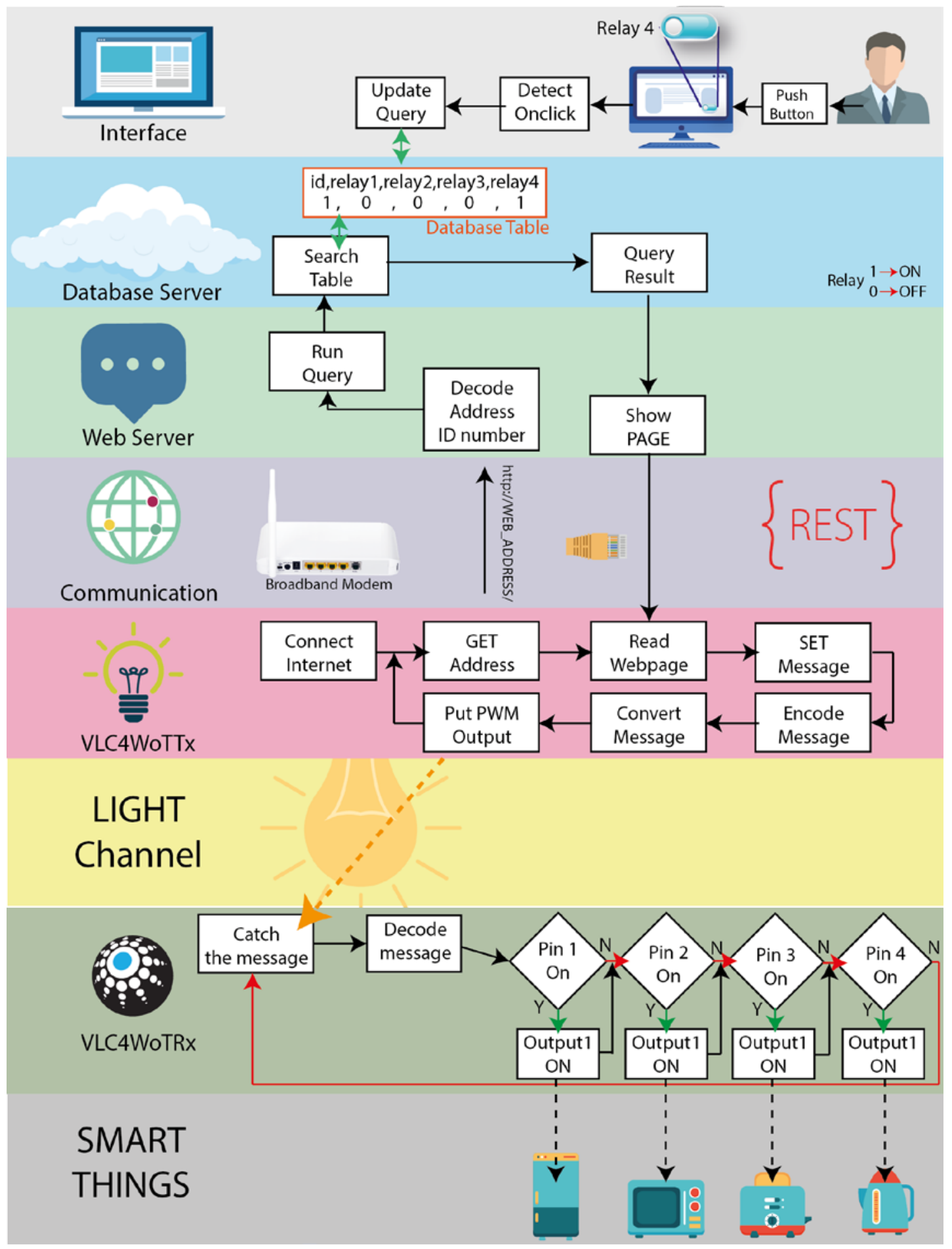

Fig. 2. VLC4WoT Software Architecture 


\subsubsection{Interface Layer}

The interface layer is a layer that allows the web server to control and monitor objects across the web. The webpage was designed dynamically and interactively. User-controlled changes on the web interface are recorded in the database. When the user wants to turn a device on or off, it applies through the interface. The result of this operation changes the value in the database. User control and management system has been developed for the interface layer. Thus, users can access their web address with their own username and password from any point.

\subsubsection{Database Server Layer}

A database server is used to manage, store and process the data of objects. Open source MYSQL is preferred for database server selection. Because MySQL is a reliable and easy to use data management system. Data of objects and relays are kept in the database. There are unique id numbers of objects. This id number is used to query the database. As a result of the query, the database fields related to the status of the relays are accessed from the table of the id number. These fields are defined as binary because they have toggle states. It is designed as 1 for True and 0 for False. If the value 0 for Relay2 from the fields is saved, it is recorded as False. This ensures that the object is closed. Since the designed object controls four different devices, four database fields have been created. In addition, a table is designed to keep track of when situations and changes are made. Thus, the changes made by the user are recorded.

\subsubsection{Web Server Layer}

Web server is a virtual machine. A domain address was appointed for access to the server. The server contains an Apache Web Server and PHP framework. It is used for querying and displaying query results in pages. Some PHP files show the request processing result on the website.

\subsubsection{Communication Layer}

The REST architecture is used in this layer for data access. Thus, objects with a microcontroller use HTTP requests to access the desired data via internet access. The VLC4WoTTX object entitled VLC4WoT1 sends the following connection request to the ip address of the server:

http://WEB_ADRESS/VLC4WoT/VLC4WoT.php?T1=VLC4IoT1

The values in the IotStatus table are first read in the cloud server after the request. The values read are written on the web page as “_id, Relay1, Relay2, Relay3, Relay4”. The data read is indicated with the "_" character at the start. This character is used for distinguishing from the html code data in the web page. As an example, the values of " $11,1,0,1,0$ " are shown for the VLC4WoT1 object. The first value denotes the id number of the VLC4WoT object. The other values indicate the active or passive states of the 4 relays. The microcontroller connects to the page and reads the values after the "_" character. With this process, time data is recorded in the VLC4WoT1 connect_time field in the IoTConn table and date is recorded in the connect_date field and $\mathrm{R}$ (Read) data is recorded in the process log field. In case of a repeated read request 
from the VLC4WoT1 object, the connect time and connect date values for the VLC4WoT1 object in the IoTConn table are updated and changed.

\subsubsection{VLC4WoTTx Layer}

The VLC4WoTTx layer is the section that transforms the data acquired via Ethernet into light data. The software has two parts. The first part is where internet connection is established, data is acquired from the cloud server which are then transmitted to the microcontroller. Whereas the second part is where the microcontroller transforms the message into binary code.

Table 1. The Frame Format for synchronization

\begin{tabular}{|c|c|c|c|c|c|}
\hline \multicolumn{7}{|c|}{ Data Drame -MaxLength 38 Chars } \\
\hline Preamble & SYNC & STX & Data & ETX & \\
\hline AA & D5 & 2 & & 3 & Hexedecimal \\
\hline 01010101 & 11010101 & 00000010 & DATA & 00000011 & Binary \\
\hline 3 & 1 & 1 & 32 & 1 & Length (Bytes) \\
\hline
\end{tabular}

The data are transmitted in a format similar to that indicated in Table1 in order to synchronize the receiver and the transmitter. The data to send (Message) is encapsulated into a frame [37]. When the transmitter wants to send a frame, it starts with a 0xAA Preamble flag to indicate that the frame is starting. Afterwords, it sends a 0xD5 SYNC flag to allow the receiver to synchronize with the clock of the transmitter. Then, 0x02 STX flag, which stands for "start-of-text", is sent to make the receiver detect a new data is upcoming; hence, DATA field takes place immediately after the STX flag. At the end, 0x03 ETX flag, which stands for "end-of-text", is sent to complete the current frame. The structure continues with this loop.

\subsubsection{LIGHT Channel Layer}

In this study, white light source was used in visible light band. Today, the white light used in interior lighting allows us to control both lighting and ambient data. The user is not influenced by the flashes since human eye does not detect the rate of flashing at high orders. Since the prototype system is located in a room in the indoor environment, control and lighting are performed together. All objects in the room are transferred data.

\subsubsection{VLC4WOTRX Layer}

The VLC4WoTTx layer is a layer that controls devices by detecting sensor data from ambient light. Fig. 3 presents a sample operating principle. VLC4WoTRx layer perceives the light data and switches the relays as either active or passive. The system continuously reads data from the light. Data is acquired when synchronization is attained. The data package is received as _1,0,1,0,1. The data snippet is separated into parts by commas. First of all, they are subject to a comparison process. Because each object has a unique id address. This enables the VLC4WoTRx objects to be used for different purposes. For example the message that starts with the "_1" character for the VLC4WoTRx object numbered 1 is put into effect in the VLC4WoTRx object numbered 1 . While the following data contains data on the relays. If "0", the relay output is designed as inactive and active if it is 1 . 

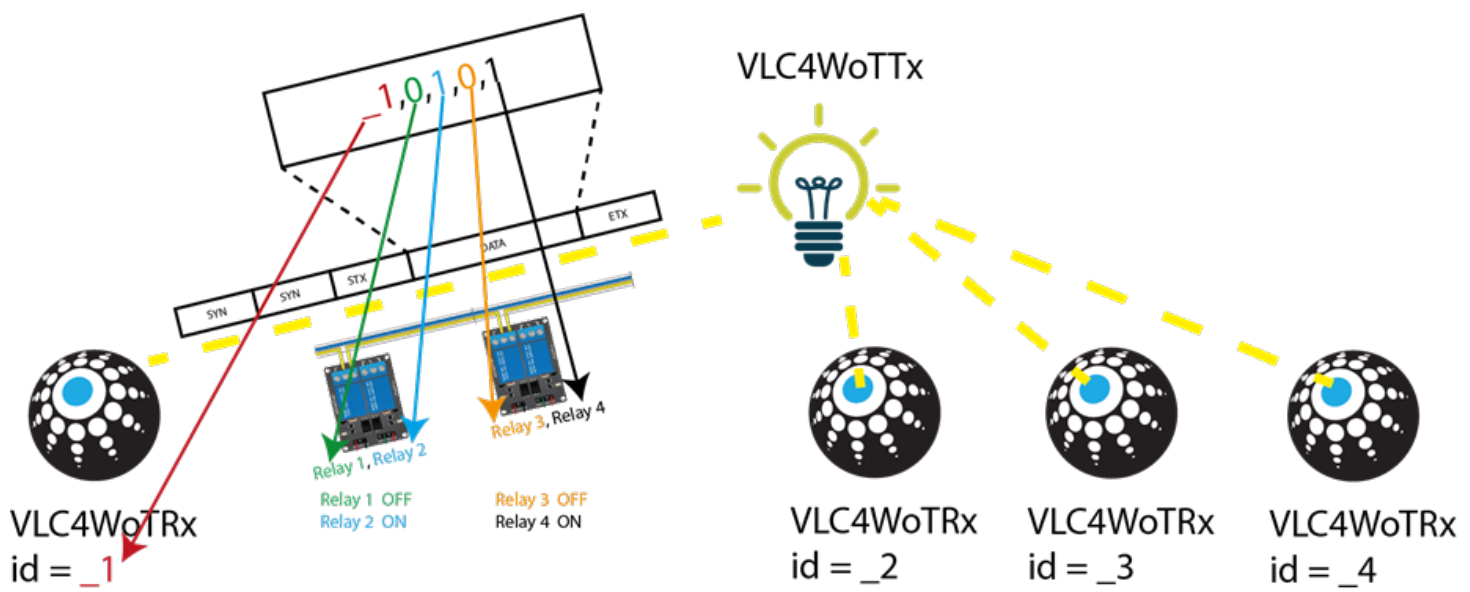

Fig. 3. Sample operating principle for the VLC4WoTRx Object

\subsubsection{Smart Things Layer}

Refers to the internal devices connected to the relay outputs of the VLC4WoTRx. Any device can be considered in indoor environments. As long as the light is on, all devices are easily controlled without the need for any communication interface.

\section{Experimental Results}

Fig. 4(a) and Fig. 4(b) show the hardware components for VLC4WoT. Fig. 4(b) is the reception unit, while Fig. 4(a) is the transmission unit. The receiving unit in Fig. 4(b) contains a quadruplet relay circuit, a microcontroller circuit and a light sensor. The relay circuit operates at a voltage of $5 \mathrm{~V}$ DC subject to whether the digital port of the microcontroller is LOW or HIGH. The TEMT 6000 light sensor connected to the microcontroller is used for reading the data transmitted by light. Electronic circuit is low cost. The transmission unit in Fig. 4(a) is comprised of three components, which are 10W COP LED, Ethernet module and microprocessor module. These components are placed inside a lamp.

An $8 \mathrm{~m}^{2}$ closed space was selected for testing the system (Fig. 5). The receiving and transmission units were placed at a distance of $2 \mathrm{~m}$ from each other. It was observed that operations between the receiver and the transmitter such as acquiring the data from the web, synchronization took place in a period of about 3 seconds.

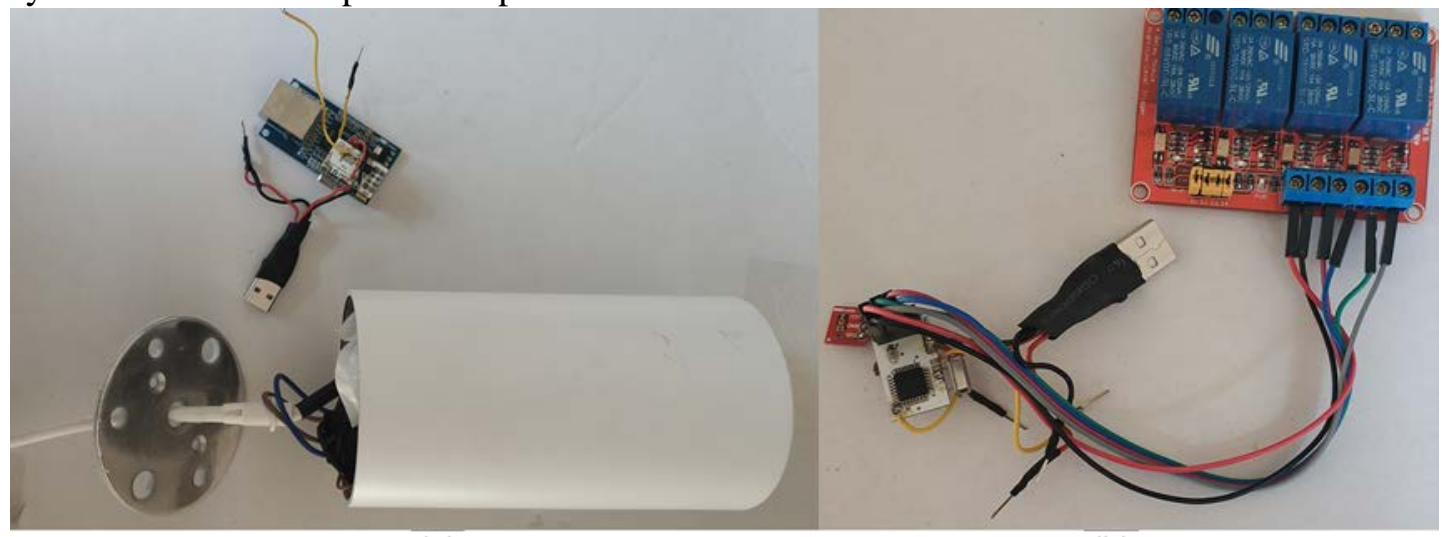

(a)

(b)

Fig. 4. The VLC4WoTRx Unit 


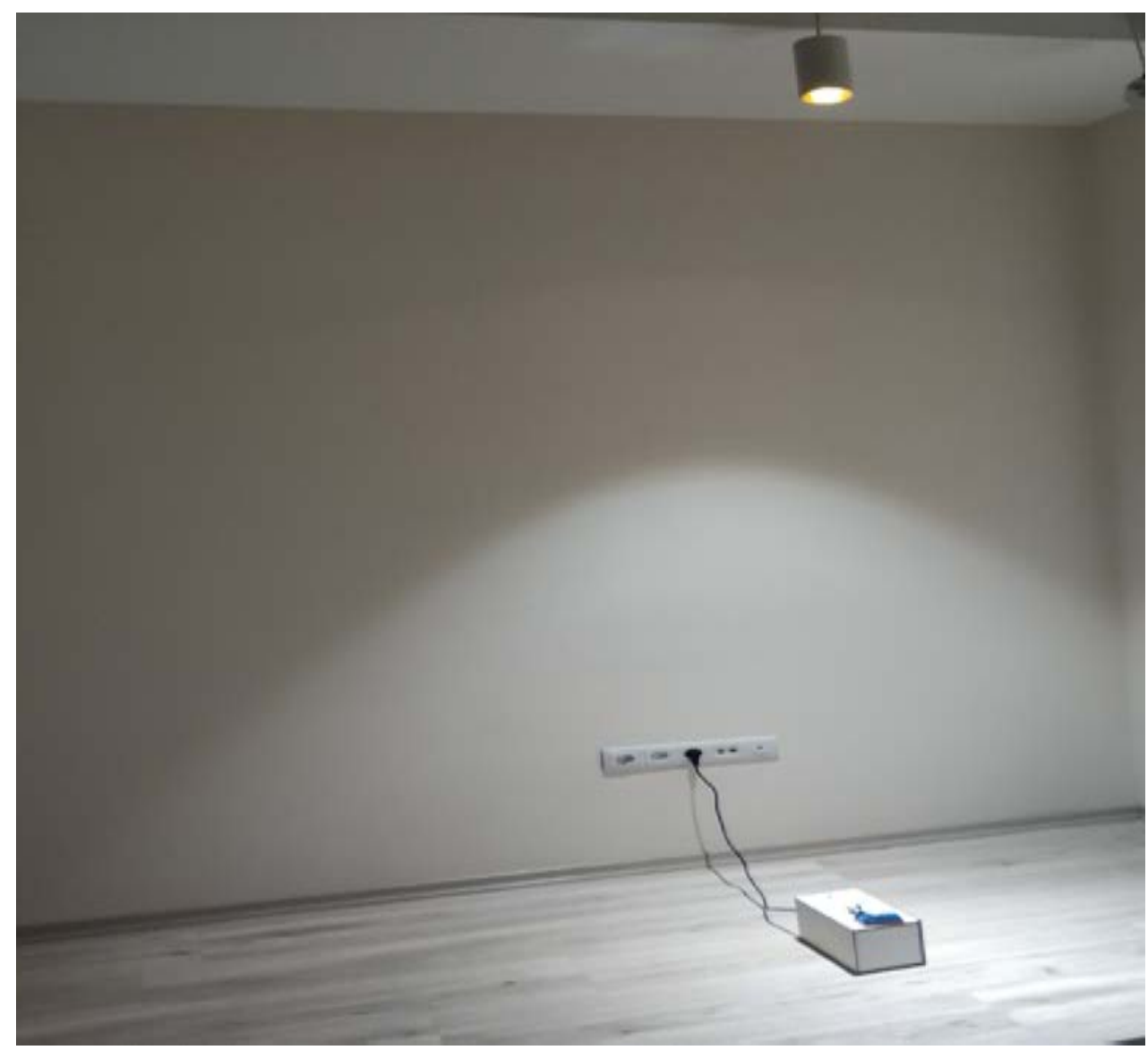

Fig. 5. The VLC4WoTTx Unit

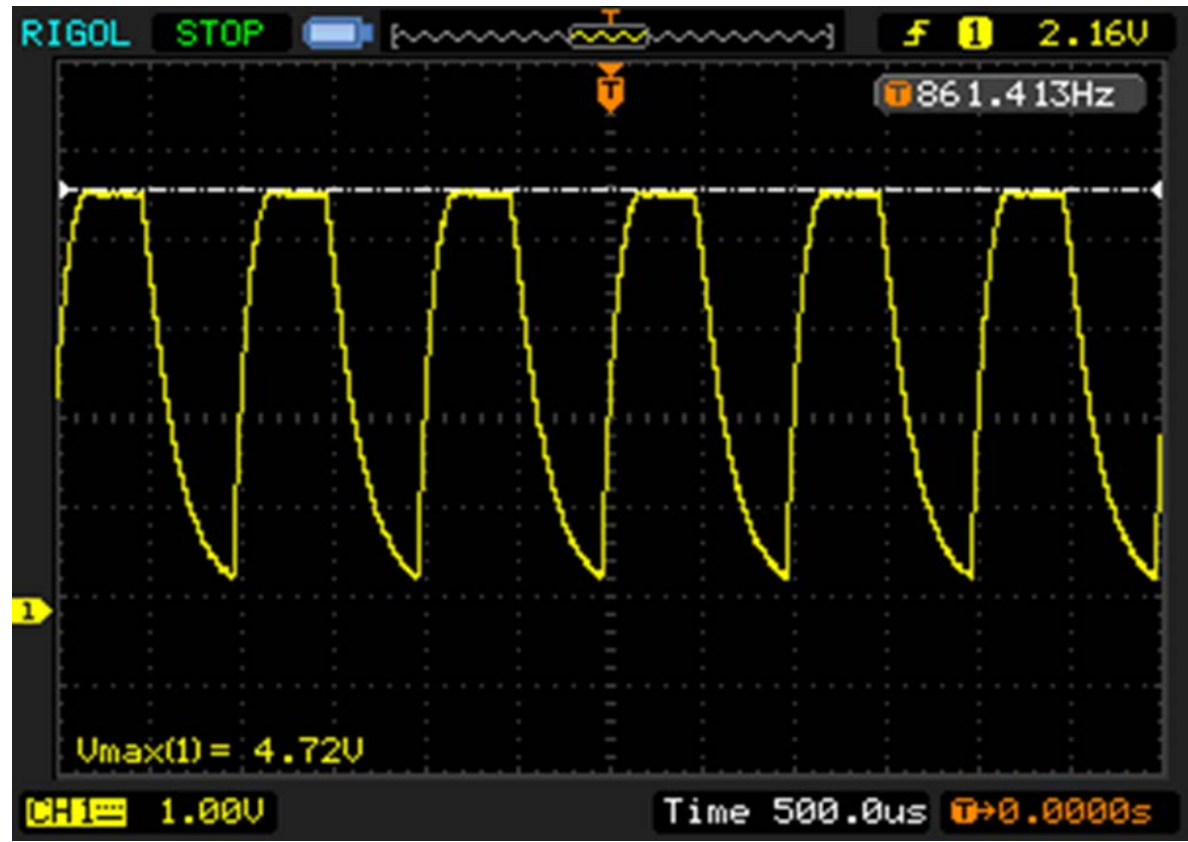

Fig. 6. The oscilloscope view for the lighting unit outlet 
Fig. 6 shows the oscilloscope view for the lighting unit outlet. As can be seen in the figure, disruptions are observed in the square signal subject to the mosfet and led driver used. However, no problem was observed in data acquisition with our trial circuit. The On-Off Keying (OOK) modulation scheme using Manchester Coding is used for data transmission.

Fig. 7 shows the screen shot for the web interface software. Fig. 7 (1) shows the Dashboard for the user. Fig. 7 (2) VLC4WoT1 control section is used to control the states of the relays. The button states in this section are continuously controlled via Ajax code. Moreover, the values in the database are updated with a JavaScript code when the button states are changed.

Fig. 7 (3) and Fig. 7 (4) shows the control buttons. In addition, these changes are recorded under the status table as well. Fig. 7 (5). The VLC4WoT1 Status section was developed for the visualization of this table. Fig. 7 (6) shows the Relay 1 screen shot for the VLC4WoT1 object. Tests were carried out during the experiment with regard to the relays turning on and off and sometimes the power was cut off to test whether they are fully operational or not. The Suspended state indicates that the VLC4WoT1 object does not operate. In this section it can be observed when Relay 1 is changed as well as the time intervals at which the device is active, inactive or inoperational.

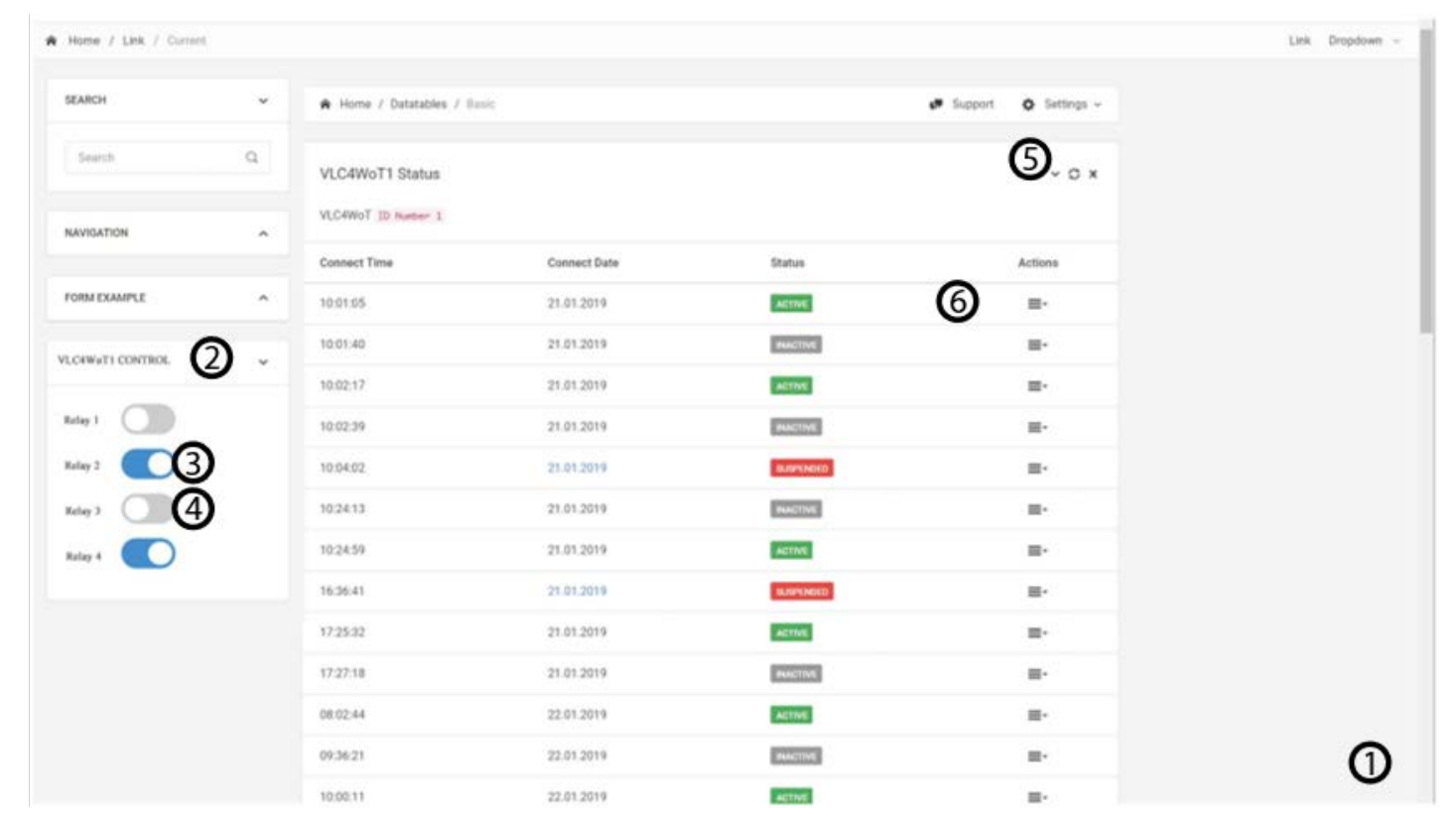

Fig. 7. Dashboard screenshot

Fig. 8 and Fig. 9 shows the example working cases. Button states that have been changed via the interface are shown in the serial screen display of the VLC4WOTTX object from the server, the serial screen display of the information received by the VLC4WOTRX object from the light, and the operation of the relays that control the internal devices. In the Fig. 8 interface environment, the positions of the buttons 2 and 4 are turned on so that relays 2 and 4 are activated from the relays. As shown in the picture, relay lights 2 and 4 indicate that the relays are active. Fig. 9 is shown in the operation of relays 1 and 4. 

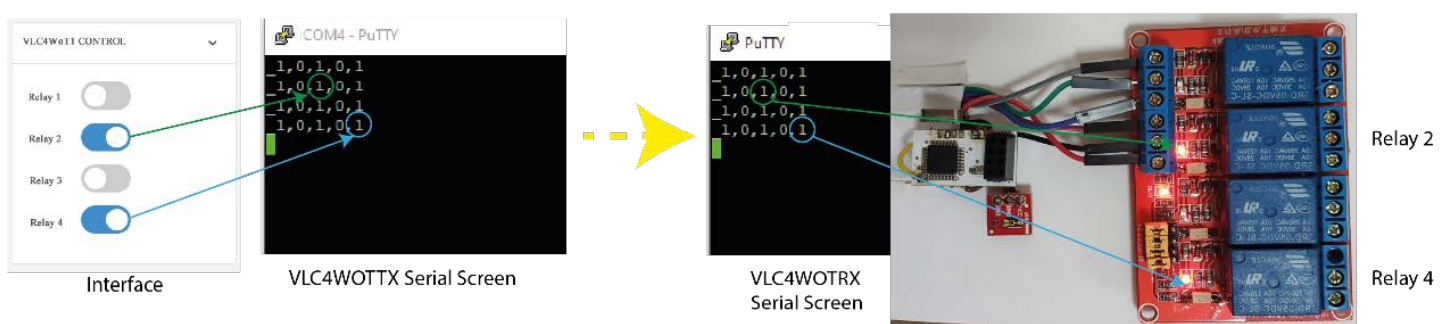

Fig. 8. Dashboard screenshot
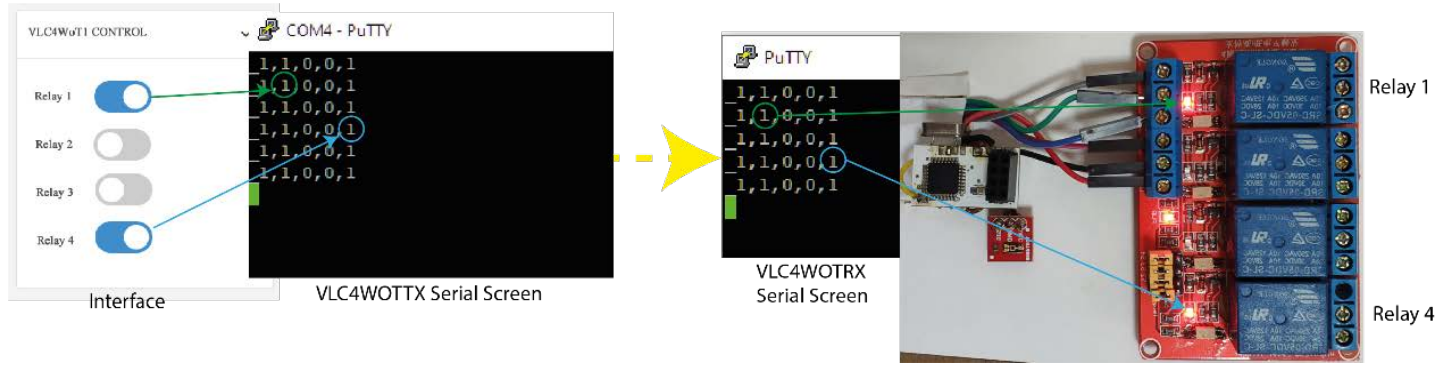

Fig. 9. Dashboard screenshot

\section{Discussion}

In this study, software and hardware platforms using VLC were presented for a good example in the web of things architecture. A basic actuator circuit that receives and processes data from the lighting unit and transmitting lighting for VLC were designed in this study. The lighting unit can be selected to have a higher light capacity or it can be differentiated subject to how it is used in internal environments. For example, all actuators inside a room can be controlled at once with a single lighting unit. Lighting units and lighting spots can be used for transmitting data to certain areas by means of directing the light. Hence, data transfer will not take place to the objects in the same environment as in the case of RF. In addition, this system can also be used for detecting the position in closed environments. The transmitting unit and circuit can be changed to transmit data at higher capacities. Accordingly, data can be transmitted to the systems requiring large data capacities such as sound or image transferring. In this way, it is possible to devise new solutions in the fields such as advertising, publicity or data flow subject to position. The web-based structure ensures that it is easily integrated into other systems.

Table 2 shows the comparison between VLC and other communication systems in indoor communication. Data rate, bandwith density, signal security, cost, installing, security, software, software installing, remote control, complexity and usability were compared. In indoor communication, Home Plug, X10, Insteon, ITU G.hn, MoCa, M-Bus, KNX, LonWorks technologies are used as wired communication. Wi-Fi, Bluetooth, $433 \mathrm{MHz}$ RF and XBee are the most commonly used wireless communication devices. In optical communication, infrared, laser and VLC systems are used. When the date rate rates are compared, VLC systems provide tremendous bandwidth in indoor communications. With Bandwidth Density, the density of the system increases in direct proportion to the spread of the system. This increase leads to greater use of bandwidth. In the comparison of Bandwidth Density, the higher the number of devices used in the "High" system stated in Table 2, the more the bandwidth is used and the "Low" shows that it uses less. A "Low" for bandwidth density in VLC systems indicates that it is advantageous. Signal Security is related to access to communication. In radio communication protocols produced by RF, radio waves are spread to 
the environment. As a result, the signal cannot be limited. As a result, RF signals can be monitored. In order to eliminate this, security has been provided by using encryption methods in RF technologies. However, since the signal can be monitored within the propagation area, communication is likely to be monitored. In optical communication, since the signal is light, the limitation of the light can be easily done in closed environments. The "high" shown in the table means that the signal security is strange. Signal security of VLC systems is high. As systems become widespread, their costs decrease. Although the use of VLC systems is not common, the cost is quite low. Installation requires technical and software information. VLC systems are enough to see the light. "Difficult" shown for in table installing means "easy" means that installation is easy. The software is only available with the semantic Web with WoT. No installation required. Because it is accessed through the internet page. "Yes" for in-table software and software installation indicates that no additional software is required and "no" for installation. No additional software and installation is required for VLC systems. VLC systems can be remotely controlled without additional hardware. Since it is accessed via the web, it can be controlled from any point where the web is accessible. This removes the limits in the remote control. In Table 2, "with extra için for remote control means additional hardware. Complexity refers to the use, installation, and adjustment of the system. The use of VLC systems is not complex compared to other systems. Usability is the degree to which users may have difficulty in operations such as web interface, software, installation, configuration. VLC systems are easier than other systems.

\begin{tabular}{|c|c|c|c|c|c|c|c|c|}
\hline 冚 & 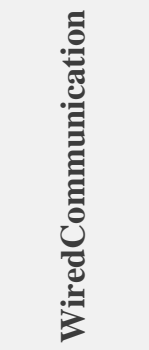 & 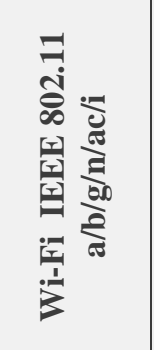 & 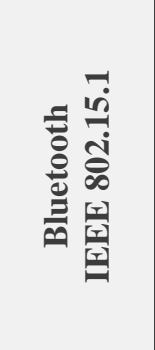 & 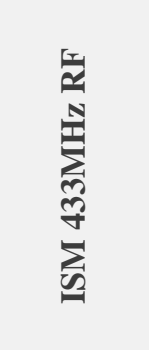 & 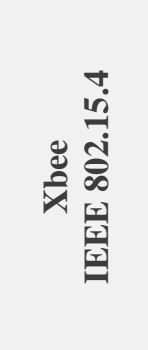 & 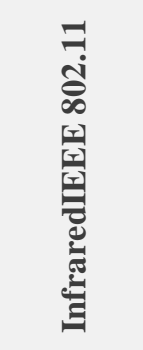 & 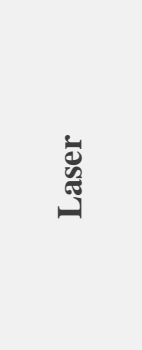 & 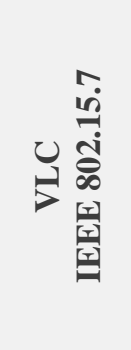 \\
\hline Data Rate & 1 Gbps & 1 Gbps & $24 \mathrm{Mbps}$ & $8 \mathrm{Kbps}$ & $250 \mathrm{Kbps}$ & $110 \mathrm{Mbps}$ & 10 Gbps & 10Gbps+ \\
\hline $\begin{array}{l}\text { Bandwidth } \\
\text { Density }\end{array}$ & High & High & High & High & Low & Low & Low & Low \\
\hline $\begin{array}{c}\text { Signal } \\
\text { Security }\end{array}$ & High & Low & Low & Low & Low & High & High & High \\
\hline Cost & $\$ 30+$ & $\$ 5+$ & $\$ 5+$ & $\$ 5^{+}$ & $\$ 30+$ & $\$ 5+$ & $\$ 30+$ & $\$ 5+$ \\
\hline Installing & Difficult & Difficult & Difficult & Difficult & Difficult & Difficult & Difficult & Easy \\
\hline Security & Good & Good & Good & Good & Good & Bad & Bad & Good \\
\hline Software & Yes & Yes & Yes & Yes & Yes & Yes & Yes & No \\
\hline $\begin{array}{l}\text { Software } \\
\text { Installing }\end{array}$ & Yes & Yes & Yes & Yes & Yes & Yes & Yes & No \\
\hline $\begin{array}{l}\text { Remote } \\
\text { Control }\end{array}$ & WithExtra & WithExtra & WithExtra & WithExtra & WithExtra & WithExtra & WithExtra & Yes \\
\hline Complexity & Complex & Complex & Complex & Complex & Complex & Complex & Complex & $\begin{array}{c}\text { Not } \\
\text { Complex }\end{array}$ \\
\hline Usability & Difficult & Difficult & Difficult & Difficult & Difficult & Difficult & Difficult & Easy \\
\hline
\end{tabular}


Even though the light sensor used in receiving unit designs is not fast enough, it is sufficient for actuators or the objects to be controlled. In the present study, the active/inactive states of the relays were examined as actuator. Devices can be connected to the relay outputs and turn on/off actions can be easily implemented. It is possible to use the light sensor in our mobile devices as receiving units in the future. Information or data transfer will take place by way of data from the light sources to our mobile devices. For example, the transmitting unit attached to the street lamp will be able to provide data such as the area it lights up, sound, position or image. Extra RF communication will not be required.

The system designed as a web software was designed with a simple interface. The system can be developed by establishing connections to the web services. Future temporal coding can be added to the software thereby enabling the system to be programmed over the web. Since the data are read from the web page, no intervention was made to the platform. However, ciphering and encryption can be added to the communication interface taking into consideration this possibility.

Studies on optical communication via infrared light and laser systems are currently ongoing as an alternative to this study. However, it has not been possible for the end users to use these devices for data transfer due to the limitations in the procurement and cost of the materials used as well as the accessibility of the environments. The fact that the receiving and transmitting sources used for VLC are easy and low-cost, that they are used for lighting and the ability to reach high data rates with the actualized communication methods make the widespread use of VLC systems possible for wireless optical communication.

\section{Conclusion}

The Light was used as a communication source for WoT architecture in this study for the internet of objects concept. Hence, an innovative and novel platform was developed that is economical, green (not harming people or the environment), energy-efficient, safe and that has not yet reached commercial popularity.

Four actuators were designed in the study as internet objects. The outputs of four different relays connected to these actuators were controlled. In addition, a lamp was designed for data transfer. These devices were controlled via a Web interface. It is considered that the presentation and actualization of a practical structure in this study will lead to novel solutions for both industrial and individual purposes in the future for the concept of the internet of objects.

\section{References}

[1] P. H. Pathak, X. Feng, P. Hu, and P. Mohapatra, "Visible Light Communication, Networking, and Sensing: A Survey, Potential and Challenges," IEEE Communications Surveys and Tutorials, vol. 17, no. 4. pp. 2047-2077, 2015. Article (CrossRef Link).

[2] C. M. Kim and S. J. Koh, "Device management and data transport in iot networks based on visible light communication,” Sensors (Switzerland), vol. 18, no. 8, p. 2741, Aug. 2018.

Article (CrossRef Link).

[3] L. U. Khan, "Visible light communication: Applications, architecture, standardization and research challenges,” Digital Communications and Networks, vol. 3, no. 2, pp. 78-88, May-2017. Article (CrossRef Link).

[4] L. Incipini, A. Belli, L. Palma, M. Ballicchia, and P. Pierleoni, "Sensing Light with LEDs: Performance Evaluation for IoT Applications,” J. Imaging, vol. 3, no. 4, p. 50, Nov. 2017. Article (CrossRef Link). 
[5] O. Ergul, E. Dinc, and O. B. Akan, "Communicate to illuminate: State-of-the-art and research challenges for visible light communications,” Physical Communication, vol. 17, pp. 72-85, Dec-2015. Article (CrossRef Link).

[6] T. Komine and M. Nakagawa, "Fundamental analysis for visible-light communication system using LED lights,” IEEE Trans. Consum. Electron., vol. 50, no. 1, pp. 100-107, Feb. 2004. Article (CrossRef Link).

[7] H. Polat, S. Oyucu, and N. Barisci, "A Study of Development of Standardised M2M Service Platform Using Restful Architecture.,” Gazi Univ. J. Sci., vol. 31, no. 2, pp. 472-487, 2018.

[8] J. Wan, D. Li, C. Zou, and K. Zhou, "M2M communications for smart city: An event-based architecture," in Proc. of 2012 IEEE 12th International Conference on Computer and Information Technology, CIT 2012, pp. 895-900, 2012. Article (CrossRef Link).

[9] L. Da Xu, W. He, and S. Li, "Internet of things in industries: A survey," IEEE Transactions on Industrial Informatics, vol. 10, no. 4. pp. 2233-2243, Nov-2014. Article (CrossRef Link).

[10] A. Pal, H. K. Rath, S. Shailendra, and A. Bhattacharyya, "IoT Standardization: The Road Ahead," Internet of Things - Technology, Applications and Standardization, InTech, 2018. Article (CrossRef Link).

[11] D. Evans, "The Internet of Things - How the Next Evolution of the Internet is Changing Everything," CISCO white Pap., no. April, pp. 1-11, 2011.

[12] S. Li, L. Da Xu, and S. Zhao, “5G Internet of Things: A survey,” J. Ind. Inf. Integr., vol. 10, pp. 1-9, Jun. 2018. Article (CrossRef Link).

[13] M. S. M. Gismalla and M. F. L. Abdullah, "Device to device communication for internet of things ecosystem: An overview,” Int. J. Integr. Eng., vol. 9, no. 4, pp. 118-123, 2017.

[14] D. Zeng, S. Guo, and Z. Cheng, "The Web of Things: A Survey (Invited Paper),” J. Commun., vol. 6, no. 6, Sep. 2011.

[15] D. Guinard, V. Trifa, and E. Wilde, “A resource oriented architecture for the web of things,” in Proc. of 2010 Internet of Things, IoT 2010, pp. 1-8, 2010. Article (CrossRef Link).

[16] M. Castro, A. J. Jara, and A. F. Skarmeta, "Enabling end-to-end CoAP-based communications for the Web of Things,” J. Netw. Comput. Appl., vol. 59, pp. 230-236, Jan. 2016. Article (CrossRef Link).

[17] S. S. Mathew, Y. Atif, Q. Z. Sheng, and Z. Maamar, "The Web of Things - Challenges and enabling technologies,” Stud. Comput. Intell., vol. 460, pp. 1-23, 2013.

Article (CrossRef Link).

[18] D. Guinard, "A Web of Things Application Architecture - Integrating the Real-World into the Web,” PhD th., ETH Zurich, no. 19891, p. 220, 2011.

[19] L. Mainetti, V. Mighali, and L. Patrono, "A software architecture enabling the web of things," IEEE Internet Things J., vol. 2, no. 6, pp. 445-454, Dec. 2015. Article (CrossRef Link).

[20] M. Kavehrad, “Sustainable energy-efficient wireless applications using light,” IEEE Commun. Mag., vol. 48, no. 12, pp. 66-73, Dec. 2010. Article (CrossRef Link).

[21] Z. Wang, Q. Wang, W. Huang, and Z. Xu, Visible Light Communications Modulation and Signal Processing. John Wiley \& Sons, 2017. Article (CrossRef Link).

[22] P. Luo, Z. Ghassemlooy, H. Le Minh, E. Bentley, A. Burton, and X. Tang, "Performance analysis of a car-to-car visible light communication system,” Appl. Opt., vol. 54, no. 7, pp. 1696-1706, Mar. 2015. Article (CrossRef Link).

[23] G. Cossu et al., "Experimental demonstration of high speed underwater visible light communications," in Proc. of the 2013 2nd International Workshop on Optical Wireless Communications, IWOW 2013, pp. 11-15, 2013. Article (CrossRef Link).

[24] N. Farr, A. Bowen, J. Ware, C. Pontbriand, and M. Tivey, “An integrated, underwater optical/acoustic communications system,” in Proc. of OCEANS'10 IEEE Sydney, OCEANSSYD 2010, pp. 1-6, 2010. Article (CrossRef Link).

[25] M. Saotome, Y. Kozawa, Y. Umeda, and H. Habuchi, "Differential-OOK System for Underwater Visible-Light Communications,” J. Signal Process., vol. 20, no. 4, pp. 175-178, 2016. Article (CrossRef Link). 
[26] H. Elgala, R. Mesleh, and H. Haas, "Indoor optical wireless communication: potential and state-of-the-art,” IEEE Commun. Mag., vol. 49, no. 9, pp. 56-62, Sep. 2011. Article (CrossRef Link).

[27] A. Zanella, N. Bui, A. Castellani, L. Vangelista, and M. Zorzi, "Internet of things for smart cities,” IEEE Internet Things J., vol. 1, no. 1, pp. 22-32, Feb. 2014. Article (CrossRef Link).

[28] C. X. Wang et al., "Cellular architecture and key technologies for 5G wireless communication networks,” IEEE Commun. Mag., vol. 52, no. 2, pp. 122-130, Feb. 2014.

Article (CrossRef Link).

[29] G. Cerruela García, I. Luque Ruiz, and M. Gómez-Nieto, "State of the Art, Trends and Future of Bluetooth Low Energy, Near Field Communication and Visible Light Communication in the Development of Smart Cities,” Sensors, vol. 16, no. 11, p. 1968, Nov. 2016.

Article (CrossRef Link).

[30] M. Novak, O. Wilfert, and T. Simicek, "Visible light communication beacon system for internet of things,” in Proc. of 2017 Conference on Microwave Techniques, COMITE 2017, pp. 1-5, 2017. Article (CrossRef Link).

[31] S. V. Tiwari, A. Sewaiwar, and Y. H. Chung, "Smart home technologies using Visible Light Communication,” in Proc. of 2015 IEEE International Conference on Consumer Electronics, ICCE 2015, pp. 379-380, 2015. Article (CrossRef Link).

[32] M. T. Niaz, F. Imdad, and H. S. Kim, "Power consumption efficiency evaluation of multi-user full-duplex visible light communication systems for smart home technologies,” Energies, vol. 10, no. 2, p. 254, Feb. 2017. Article (CrossRef Link).

[33] C. M. Kim, S. Il Choi, and S. J. Koh, "IDMP-VLC: IoT device management protocol in visible light communication networks," in Proc. of International Conference on Advanced Communication Technology, ICACT, pp. 578-583, 2017. Article (CrossRef Link).

[34] S. Rajagopal, R. Roberts, and S.-K. Lim, "IEEE 802.15.7 visible light communication: modulation schemes and dimming support,” IEEE Commun. Mag., vol. 50, no. 3, pp. 72-82, Mar. 2012. Article (CrossRef Link).

[35] A. Jovicic, J. Li, and T. Richardson, "Visible light communication: opportunities, challenges and the path to market,” IEEE Commun. Mag., vol. 51, no. 12, pp. 26-32, Dec. 2013.

Article (CrossRef Link).

[36] S. Rajbhandari et al., "High-Speed Integrated Visible Light Communication System: Device Constraints and Design Considerations,” IEEE J. Sel. Areas Commun., vol. 33, no. 9, pp. 1750-1757, Sep. 2015. Article (CrossRef Link).

[37] B. B. Purkayastha and K. K. Sarma, "Synchronization," A Digital Phase Locked Loop based Signal and Symbol Recovery System for Wireless Channel, Springer, pp. 127-151, 2015. Article (CrossRef Link). 


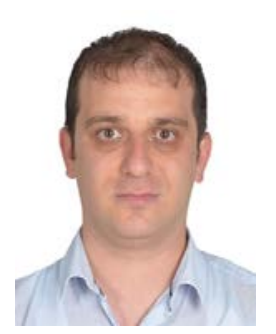

MAHMUT DURGUN received the B.E. degree in computer engineering from Karadeniz Technical University, Turkey, in 2011, and M.S. degrees in Mechatronics Engineering from Tokat Gaziosmanpasa University Turkey, in 2014. The Ph.D. degree in Mechatronics Engineering from Tokat Gaziosmanpasa University Turkey, Tokat, in 2019. He is currently working as a Assistant Professor with the Department of Mechatronics Engineering, Tokat Gaziosmanpasa University, Turkey. His research interests include visible light communication, wireless sensor networks, wireless communication, and the IoT-based services.

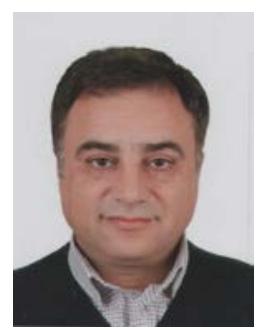

LEVENT GÖKREM was born in Tokat, TURKEY in 1970. He received the B.S. degree in Department of Electronics and Computer Education from Gazi University, Turkey, in 1993. He received the M.S. degree in Electronics and Computer Education from the Sakarya University Turkey, in 2003 and the Ph.D. degree in Industrial Technology Education from Gazi University, Ankara, in 2009. He has been an Assistant Professor with the Department of Mechatronics Engineering, Tokat Gaziosmanpasa University, Turkey. His research areas are Internet of Things, Image processing and wireless communication. 